\title{
Limiting current in a relativistic diode under the condition of magnetic insulation
}

\author{
Mike Lopez, Y. Y. Lau, John W. Luginsland, ${ }^{\text {a) }}$ David W. Jordan, \\ and Ronald M. Gilgenbach \\ Department of Nuclear Engineering and Radiological Sciences, University of Michigan, Ann Arbor, \\ Michigan 48109-2104
}

(Received 20 February 2003; accepted 1 August 2003)

\begin{abstract}
The maximum emission current density is calculated for a time-independent, relativistic, cycloidal electron flow in a diode that is under the condition of magnetic insulation. Contrary to conventional thinking, this maximum current is not determined by the space charge limited condition on the cathode, even when the emission velocity of the electrons is assumed to be zero. The self electric and magnetic fields associated with the cycloidal flow are completely accounted for. This maximum current density is confirmed by a two-dimensional, fully electromagnetic and fully relativistic particle-in-cell code. (C) 2003 American Institute of Physics. [DOI: 10.1063/1.1613654]
\end{abstract}

\section{INTRODUCTION}

Magnetic insulation remains an important problem in pulse power systems and high power microwave sources. While there have been numerous publications on the subject in the last three decades, ${ }^{1-19}$ strictly speaking, the maximum injected current for a time-independent cycloidal flow in a relativistic diode under the condition of magnetic insulation has never been solved, even for the one-dimensional, planar geometry. The present paper provides the solution to this fundamental problem.

When a diode is magnetically insulated, an electron released from the cathode begins its cycloidal trajectory under the crossed electric and magnetic field, reaching a maximum excursion within this crossed-field gap before returning to the cathode surface (Fig. 1). A collection of such electrons then constitutes a cycloidal electron flow in the gap. In this paper we calculate the maximum emission current density that is allowed under the time-independent condition, for a given gap voltage, gap spacing, and insulating magnetic field.

It has often been taken for granted that the maximum current that can be admitted into a gap is attained when there is sufficient space charge in the gap to force the electric field on the emitting surface equal to zero. Under this condition, additional electrons with zero emission velocity will be returned to the cathode and a virtual cathode is formed. ${ }^{20-22}$ This condition of zero surface electric field is known as the space charge limited (SCL) condition. In a nonmagnetized diode, the maximum emission current density is indeed given by the SCL condition for electrons with zero emission velocity. Adopting such an assumption for a magnetically insulated gap is natural. Thus, Lovelace and $\mathrm{Ott}^{2}$ calculated the equilibrium relativistic cycloidal flows in a magnetically insulated gap under the SCL assumption. They included the self-electric and magnetic fields of the cycloidal flow. From the discussions given at the beginning of this paragraph, one

${ }^{a)}$ Science Applications International Corporation, Albuquerque, NM. may be tempted to take this SCL solution of Lovelace and $\mathrm{Ott}^{2}$ as the maximum emission current density for timeindependent cycloidal flows in a relativistic crossed-field gap.

Surprisingly, Christenson ${ }^{3}$ discovered that the maximum emission current density was not given by the SCL condition for a deeply nonrelativistic cycloidal crossed-field flow, even if the electrons are emitted with essentially zero velocity. She discovered this unexpected result in her simulation study of noise in crossed-field flows. She found that the maximum allowable current was slightly higher than that predicted from the SCL condition. This result is unmistaken, even though the code she used, PDP $1,{ }^{23}$ is a one-dimensional (1D), electrostatic code that ignores relativistic and diamagnetic effects. When the SCL condition is relaxed, the maximum current is derived semianalytically in the nonrelativistic regime and there is excellent agreement between the analytic theory and the simulation results. ${ }^{3}$

In this paper, we present the maximum emission current density in a relativistic, magnetically insulated diode. Motivated by the findings reported in Christenson, ${ }^{3}$ we do not use the SCL condition. Instead, we determine the surface electric field that allows maximum emission current density. The analysis is otherwise similar to Ott and Lovelace, ${ }^{2}$ including the relativistic effects and the effects of the self electric and magnetic field of the cycloidal electron flow. While Ron, Mondelli, and Rostoker ${ }^{1}$ also considered a relativistic diode under the condition of magnetic insulation, they left the electric field and magnetic field on the cathode surface as free parameters. Mendel ${ }^{9}$ considered the general case of arbitrary canonical angular momentum, but much of his analysis also left the surface electric field as a free parameter. Here we determine the surface electric field (and surface magnetic field) that yields the maximum injection current density, using the model and notations of Lovelace and $\mathrm{Ott}^{2}$ Our results are in the form of a set of universal curves that give the 


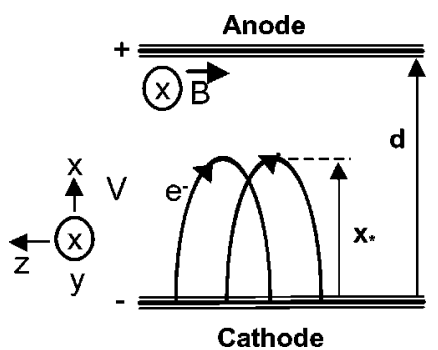

FIG. 1. Cycloidal electron orbits in a magnetically insulated crossed-field gap.

maximum current density at various gap voltages, while the gap spacing and the external magnetic field may have general values as long as the condition of magnetic insulation is satisfied. These curves reduce to the established results in the deeply nonrelativistic regime. They have been confirmed by a two-dimensional particle-in-cell code, which is fully relativistic and fully electromagnetic. It is interesting to note that the maximum current density in a magnetically insulated diode is, in general, about $10 \%$ higher than that predicted from the SCL condition, though the physical reason remains unclear.

\section{THEORY AND SIMULATION}

Consider a planar gap with the cathode located at $x=0$ and the anode at $x=d$. An external magnetic field, $B$, is imposed in the $y$-direction (Fig. 1). The cathode is held at zero potential and the anode is held at a positive voltage, V. Electrons are injected from the cathode with zero emission velocity. They reach a maximum height, $x_{*}$, into the gap before returning to the cathode $\left(x_{*}<d\right)$. We shall determine the maximum injection current density, $J_{+}$, for this timeindependent cycloidal flow. Following Lovelace and Ott, ${ }^{2}$ we assume that the total magnetic flux within the gap is unchanged regardless of the injected current density. This corresponds to perfectly conducting cathode and anode plates. Mathematically, this assumption removes the arbitrary constant when we solve for the magnetic field $\mathbf{H}$ from the Ampère's law, curl $\mathbf{H}=\mathbf{J}$. Thus, the total magnetic flux is equal to that of the vacuum gap. We shall also follow Lovelace and Ott to use the dimensionless quantities,

$$
\begin{aligned}
& \bar{V}=1+\frac{e V}{m c^{2}}, \\
& \eta=\omega_{c} d / c,
\end{aligned}
$$

to represent the gap voltage and the external magnetic field. In Eqs. (1) and (2), $e(e>0)$ and $m$ are, respectively, the electron charge and rest mass, $c$ is the light speed, and $\omega_{c}$ $=e B / m$ is the nonrelativistic electron cyclotron frequency associated with the external magnetic field. SI units are used throughout.

Magnetic insulation requires that

$$
\frac{B}{B_{\mathrm{HR}}} \equiv \frac{\eta}{\sqrt{\bar{V}^{2}-1}}>1,
$$

i.e., the external magnetic field, $B$, exceeds the relativistic Hull cutoff magnetic field, $B_{\mathrm{HR}}$. The latter is determined from Eq. (3) by replacing the $(>)$ sign with the $(=)$ sign. The magnitude of the emission current density, $J_{+}$, in units of the $1 \mathrm{D}$ nonrelativistic Child-Langmuir value $J_{\mathrm{CL}}$, may be written as

$$
\frac{J_{+}}{J_{\mathrm{CL}}}=(k d)^{2}\left(\frac{9}{8 \sqrt{2}}\right) \frac{1}{(\bar{V}-1)^{3 / 2}},
$$

where $J_{\mathrm{CL}}=(4 / 9) \varepsilon_{0}(2 e / m)^{1 / 2} V^{3 / 2} / d^{2}$ and $k$ is defined by $J_{+}=\left(m c^{3} / e\right)\left(\varepsilon_{0} / 2\right) k^{2}$, as in Ref. 2 . Note that $k$ has the unit of $m^{-1}$. In this paper, we seek to maximize the value of Eq. (4) as a function of $B / B_{\mathrm{HR}}$ at various values of gap voltage, $V$.

The analysis is substantially more complicated when the SCL condition is relaxed for the present problem. In this paper, we shall only record the few equations that are needed to determine the maximum current density. They are chosen so that the relevant physical quantities (e.g., the thickness of the electron sheath, the degree of diamagnetism, etc.) may be obtained. They are written in such a way that they may readily be compared with the corresponding ones of Lovelace and $\mathrm{Ott}^{2}$ when the SCL condition is taken. The notations are also similar.

Following Lovelace and Ott, we first introduce two dimensionless parameters, $\alpha$ and $\beta$, defined as the ratio of the total magnetic field to the external magnetic field, at $x=0$ and at $x=x_{*}$ respectively. Here, $x_{*}$ is the maximum excursion of an electron into the gap before it starts to return to the cathode and the total magnetic field includes the diamagnetic field. Another dimensionless parameter, $\kappa$, is defined:

$$
\kappa=\left(\frac{2 k d}{\eta \alpha}\right)^{4}
$$

We now introduce the dimensionless surface electric field, $\delta$, defined as

$$
\delta^{2}=\left(\frac{E_{s} d}{m c^{2} / e}\right)^{2}\left(\frac{1}{\eta \alpha}\right)^{2}
$$

where $E_{s}$ is the magnitude of surface electric field. We take $\delta$ to be non-negative. The value of $\delta$ needs to be determined to maximize $J_{+} / J_{\mathrm{CL}}$.

The next seven equations, Eqs. (7)-(13), are obtained from the time-independent force law, continuity equation, Ampère's law, and Poisson equation. Their $\delta=0$ limits are identical to the corresponding ones of Lovelace and Ott, ${ }^{2}$ which we shall individually identify. Let us first introduce a function,

$$
f(\rho)=\frac{\kappa^{1 / 2}}{2} \sqrt{\rho^{2}-1}-\left(1-\frac{1}{\rho^{2}}\right)+\delta^{2},
$$

whose root $\rho_{m}=\rho_{m}(\kappa, \delta)$ is to be determined numerically in terms of $\kappa$ and $\delta$ by solving $f\left(\rho_{m}\right)=0$. Among the multiple roots of Eq. (7), we choose only the one that gives the same $\rho_{m}$ as in Lovelace and Ott in the limit $\delta$ approaching zero. 
[In the limit $\delta=0$, Eq. (7) appears in the integrand of Eq. (9) of Lovelace and Ott. $\left.{ }^{2}\right]$ From this root of $\rho_{m}$, we may define the function,

$$
\theta_{*}(\kappa)=2 \int_{1}^{\rho_{m}} \frac{d \rho}{\rho^{2} \sqrt{f(\rho)}} .
$$

The physical meanings follow: $\rho=\gamma / \gamma_{z}=(1$ $\left.+\gamma^{2} \beta_{x}^{2}\right)^{1 / 2}$, where $\beta_{x}$ and $\beta_{z}$ are the velocity components (in units of the light speed $c$ ) in the $x$ and $z$ direction respectively, $\gamma=\left(1-\beta_{x}^{2}-\beta_{z}^{2}\right)^{-1 / 2}$ is the relativistic mass factor, $\gamma_{z}=\left(1-\beta_{z}^{2}\right)^{-1 / 2}$ is the relativistic mass factor associated with the $z$-component of the velocity (Fig. 1), and $f(\rho)$ $=[(d / \eta \alpha) d \rho / d x]^{2}$. As in Lovelace and Ott, $\gamma=\rho \cosh \theta$, and the vector potential (normalized to $m c^{2} / e$ ) associated with the self-consistent magnetic field is $\bar{A}=\rho \sinh \theta$. At $x$ $=x_{*}, \theta=\theta_{*}$, and $\rho=1$. Thus, the electron's relativistic mass factor at $x_{*}$ is equal to $\cosh \theta_{*}$ and the electrostatic potential at $x_{*}$ is equal to $\left(m c^{2} / e\right)\left(\cosh \theta_{*}-1\right)$, by energy conservation. Moreover, at $x=x_{*} / 2, \rho=\rho_{m}$.

The value of $x_{*}$ is given by

$$
\begin{aligned}
& \frac{x_{*}}{d}=\frac{g(\kappa)}{\eta \alpha}, \\
& g(\kappa)=2 \int_{1}^{\rho_{m}} \frac{d \rho}{\sqrt{f(\rho)}} .
\end{aligned}
$$

It may be shown that the following equations are satisfied:

$$
\begin{aligned}
& \bar{V} \cosh \theta_{*}-\eta \sinh \theta_{*}-1=\delta\left(\bar{V} \sinh \theta_{*}-\eta \cosh \theta_{*}\right), \\
& \beta / \alpha=\cosh \theta_{*}-\delta \sinh \theta_{*} \\
& \eta-\sinh \theta_{*}=\eta \beta-\left(\cosh \theta_{*}-\delta \sinh \theta_{*}\right) g(\kappa) .
\end{aligned}
$$

Equations (8), (10), and (12) may readily be compared with, respectively, Eqs. (11a), (9), and (21) of Ref. 2. Equations (13) and (11) may also be compared, respectively, with Eqs. (12b) and (12a) of Ref. 2 if one recalls that the relativistic factor $\left(\bar{V}_{*}\right)$ at $x=x_{*}$ is $\cosh \theta_{*}$ and that the normalized vector potential $\left(\bar{A}_{*}\right)$ there is $\sinh \theta_{*}$.

The following algorithm has been used to determine the limiting current for a pair of assigned values, $(\eta, \bar{V})$, that satisfies Eq. (3). The value of $B / B_{\mathrm{HR}}$ is immediately known from Eq. (3) for this pair of normalized gap voltage and external magnetic field. The following steps are then taken:

(a) Assign a value of $\delta(\delta>0)$.

(b) Obtain numerically the solution to $\theta_{*}$ from Eq. (11). The meaningful solution is the one that is the same as in Ref. 2 in the limit $\delta=0$ (SCL).

(c) Find the value of $\kappa$ so that Eq. (8) yields the same value of $\theta_{*}$.

(d) For these values of $\delta$ and $\kappa$, obtain $g(\kappa)$ from Eq. (10).

(e) Obtain $\beta$ from Eq. (13) and then $\alpha$ from Eq. (12).

(f) Obtain $k d$ from Eq. (5) and hence $J_{+} / J_{\mathrm{CL}}$ from Eq. (4).

(g) Repeat Step (a) until $J_{+} / J_{\mathrm{CL}}$ is maximized with respect to $\delta$.

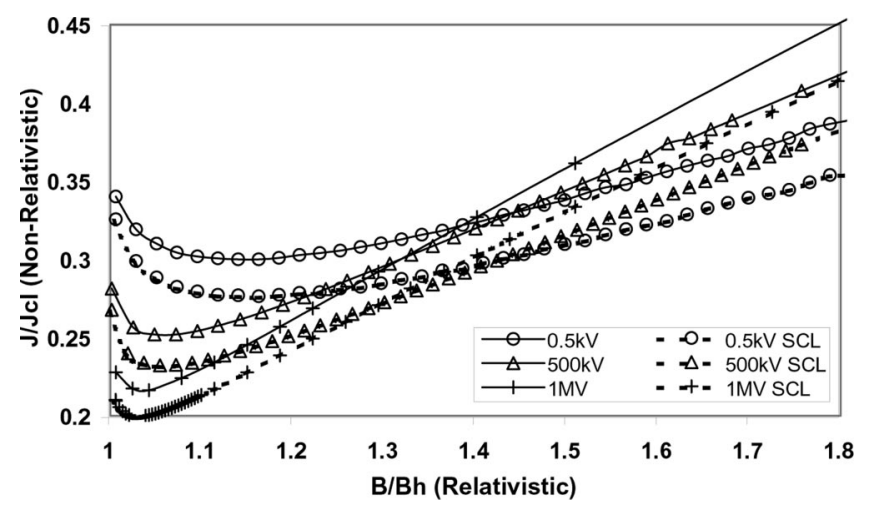

FIG. 2. The normalized limiting current density in a crossed-field gap under the condition of magnetic insulation, at various gap voltage, V. Also shown are the corresponding values when the space charge limited (SCL) condition is imposed on the cathode.

Figure 2 shows the normalized limiting current density at three diode voltages, $V=0.5 \mathrm{kV}, 500 \mathrm{kV}$, and $1 \mathrm{MV}$, determined from the algorithm given in the preceding paragraph. Also shown are the values when the SCL condition is imposed. The results of Lovelace and $\mathrm{Ott}^{2}$ are indistinguishable from the curves in Fig. 2 under the SCL condition. It is seen that the true maximum injection current density is higher than that obtained from the SCL condition by about $10 \%$. From Fig. 2, higher gap voltage seems to allow a lower current into the gap, in particular at lower values of the magnetic field, $B$. This seemingly counterintuitive result arises from our normalization of $J_{+}$by the non-relativistic ChildLangmuir value, $J_{\mathrm{CL}}$, as was done in Lovelace and Ott. ${ }^{2}$ At relativistic energies, Jory and Trivelpiece ${ }^{24}$ show from an electrostatic, 1D analysis that their 1D current density scales as $V$ instead of $V^{3 / 2}$. We should emphasize that the 1D analysis of Jory and Trivelpiece ${ }^{24}$ completely ignores the self magnetic field, which for a high current relativistic diode may exert a force that is comparable to the space charge force. The limiting current density including the selfmagnetic field is very difficult to calculate whenever $B$ $<B_{\mathrm{HR}}$, and we shall return to this unsolved problem toward the end of this paper.

The calculations given above have been confirmed by three tests: (a) We have shown that our numerical algorithm yields identical results to Lovelace and $\mathrm{Ott}^{2}$ if we set the surface electric field equal to zero, as stated in the preceding paragraph. (b) The $V=0.5 \mathrm{kV}$ curve in Fig. 2 (the one without the SCL assumption) is indistinguishable from Christenson's nonrelativistic results, ${ }^{3}$ the latter were obtained by an entirely different algorithm. (c) Most importantly, we use the simulation code, MAGIC, ${ }^{25}$ which is a $2 \mathrm{D}$, fully relativistic and fully electromagnetic code to show that steady state cycloidal flow can occur beyond the injection current predicted from the SCL condition, and that the maximum injection current is consistent with the formulation given above. The data of sample MAGIC runs are shown in Fig. 3, where we increase the injection current in a $500 \mathrm{kV}$ diode. Time independent flow ceases to exist when the injection current reaches the level predicted in our theory.

In the above-mentioned MAGIC simulations, the gap 


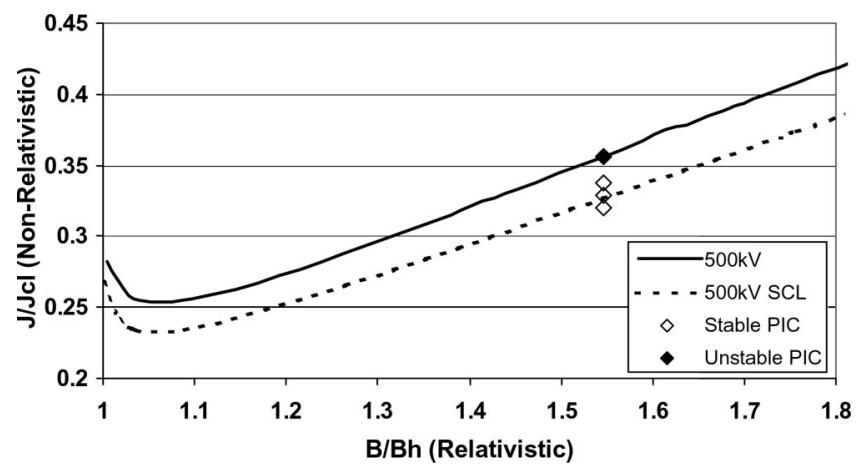

FIG. 3. MAGIC simulation data for a $500 \mathrm{kV}$ diode. The two curves are reproduced from Fig. 2, with the upper curve representing the maximum injection current, and the lower curve assuming space charge limited condition on the cathode. Virtual cathode is observed in the MAGIC simulation only when the injected current reaches the upper curve.

separation is $0.0025 \mathrm{~m}$. The width of the parallel plates is $0.025 \mathrm{~m}$. The left and right sides of the simulations are closed with a periodic boundary condition. The voltage is applied between the two plates with an external magnetic field in the ignorable direction. Since these are 2D Cartesian simulations, the current density is in units of $\mathrm{A} / \mathrm{m}$ as the ignorable direction is unit meter length by default. The injected current density is imposed at the surface in units of $\mathrm{A} / \mathrm{m}$ per unit length in the ignorable direction. This is translated into charge on a given number of macroparticles per cell. Typical emission numbers are 2 to 8 macro-particle per cell per time step. The particles are injected into the simulation with an energy of $1 \mathrm{eV}$. The injected current is ramped over $4 \mathrm{~ns}$ to avoid shock excitation of the system. It should be noted that it is critical to avoid shock excitation. Raising the current too rapidly, or using an insufficient number of particles (i.e., discrete particle noise) can cause collapse of the flow into turbulence in just a few cyclotron periods.

It is not obvious why the limiting current, as represented by the solid curves in Figs. 2 and 3, should be about $10 \%$ higher than that associated with the SCL condition, represented by the dotted curves in Figs. 2 and 3. Since this is also true for the nonrelativistic diode, as shown in the $0.5 \mathrm{kV}$ curves in Fig. 2, the self-magnetic field is not the main reason why the limiting current should be higher than that predicted from the SCL condition. In fact, the evolution of the phase space plots in our particle simulations behaves qualitatively the same for both a relativistic diode and a nonrelativistic diode, as we increase the injected current from zero to a value beyond the (theoretically predicted) limiting current. To avoid the complications associated with the selfmagnetic field, we describe in the next paragraph this evolution for just a deeply nonrelativistic crossed-field diode, which has been documented in great detail in Ref. 3 .

In a nonrelativistic diode under magnetic insulation, as the injection current increases, the phase space plot ( $x$ vs $v_{x}$, see Fig. 1) remains qualitatively the same as that of a single particle in the vacuum gap, up to the predicted limiting current. The electric field on the cathode surface is always accelerating for all values of injection current below the predicted limiting current. That is, in the simulation, when the injection current equals the value given by the SCL condition (the dotted curves in Fig. 2), the surface electric field on the cathode is not equal to zero. In fact, that surface electric field never equals zero as long as the injected current is below the predicted limiting current (which is typically 10\% higher than the corresponding dotted curve in Fig. 2). While the limiting current density is about $10 \%$ higher than that corresponding to the SCL condition, the total charge in the gap at this limiting current density is less than that corresponding to the SCL condition; the latter is also equal to the total space charge in the Brillouin flow of the same external electric and magnetic fields. [For this deeply nonrelativistic regime, it can readily be shown that, when the SCL condition is assumed, the maximum excursion of the cycloidal orbit $\left(x_{*}\right.$, Fig. 1) is identical to the Brillouin hub height for the same magnetic field and gap voltage, and that the total space charge in that cycloidal flow is also identical to that of the Brillouin flow.] Once the injection current exceeds the predicted limiting value, the laminar cycloidal flow quickly collapses into a mildly turbulent Brillouin flow (1) which has little $x$-directed motion, (2) whose electric field on the surface is close to zero, and (3) whose space charge at the gap is roughly given by that given in the laminar Brillouin flow.

Thus, for a fixed value of $B / B_{H}(>1)$, at the value of $J / J_{\mathrm{CL}}$ on a dotted curve of Figs. 2 and 3 , there are two solutions, one with the surface electric field $E_{s}$ equal to zero (SCL condition) and the other with $E_{s}$ nonzero. All simulations so far suggest that the former is inaccessible. We shall postpone to a separate publication for an in depth examination of the multiplicity of the solutions, the seemingly inaccessibility of the SCL solution, and the implications on the numerical algorithms that imposed such a condition on the emitting surface.

\section{REMARKS}

We should point out that the cycloidal flow solutions studied in this paper are likely to be unstable. In previous 1D simulations of nonrelativistic, cycloidal crossed-field flows using the electrostatic code PDP $1,{ }^{23}$ it is found that a small ac gap voltage, ${ }^{3,26}$ or a small external resistance, ${ }^{27}$ or a small misalignment in the external magnetic field, ${ }^{28}$ may render the cycloidal flow unstable even when the emission current density is only a small fraction of the critical values depicted in Fig. 2. The final state of these destabilized cycloidal flows, to a high degree, is approximated by the Brillouin flow. This final Brillouin state is in fact anticipated by Slater ${ }^{29}$ and by Buneman, ${ }^{30}$ who argue that the equilibrium cycloidal flow is at a higher energy state than the equilibrium Brillouin flow, as the latter does not possess $x$-directed motion (Fig. 1). In spite of the overwhelming evidence in favor of the Brillouin state, McDowell ${ }^{31}$ recently concluded from his simulations that the final state in a crossed-field device might well be the cycloidal orbits that are studied by Slater after all, with an electron density extending much further into the crossedfield gap than is allowed by the Brillouin layer. In this sense, the likely final state of the crossed-field flow remains an open question. Relativistic Brillouin flows are studied in Refs. 32, 33. 
If the electron pulse length is sufficiently short, neither the time-independent flow solution studied in this paper nor the Brillouin state is developed. The emission current density in a relativistic diode that is magnetically insulated may then be substantially higher than that predicted in this paper for the time-independent solution. This conjecture is based on a recent study of a nonmagnetized, laser-triggered diode in the deeply nonrelativistic regime, ${ }^{34}$ where the emission current density may be several times the classical Child-Langmuir value in a short, transient electron bunch.

The analysis given in this paper is restricted only to a magnetically insulated gap, where $B>B_{\mathrm{HR}}$. For $B<B_{\mathrm{HR}}$, the electrons will reach the anode, in which case the limiting current density in the relativistic diode is very difficult to formulate. The electron velocity necessarily contains all three $(x, y, z)$ components when one includes the selfmagnetic field. The self-magnetic field itself is rather complicated because it is generated by two current components: one parallel to the cathode and the other perpendicular to the cathode (electrons crossing the gap). In fact, it must have a two-dimensional dependence in $(x, z)$. This problem, while difficult to attack analytically, is of considerable interest to the study of the magnetically insulated line oscillator $\left(\mathrm{MILO}^{35-39}\right)$, a high power microwave source in which the electron flow is determined by its self-magnetic field (instead of the external magnetic field, which is absent in the MILO). In the deeply nonrelativistic regime, where the self-magnetic field can safely be ignored, the limiting current for a noninsulated gap has been calculated. ${ }^{3,17,18,40}$ In this case, the SCL condition applies, even to electron emission that is restricted spatially $^{41-43}$ and temporally. ${ }^{34}$

\section{ACKNOWLEDGMENTS}

We thank Dr. L. Ludeking for assistance with the MAGIC code.

This work was supported by AFOSR Grant No. F4962002-1-0089 and DUSD (S\&T) under the Innovative Microwave Vacuum Electronics MURI Program, managed by the Air Force Office of Scientific Research under Grant No. F49620-99-1-0297, and by the Northrop Grumman Industrial Associates Program.

${ }^{1}$ A. Ron, A. A. Mondelli, and N. Rostoker, IEEE Trans. Plasma Sci. PS-1, 85 (1973).

${ }^{2}$ R. V. Lovelace and E. Ott, Phys. Fluids 17, 1263 (1974).

${ }^{3}$ P. J. Christenson, Ph.D. dissertation, University of Michigan, Ann Arbor (1996).

${ }^{4}$ J. A. Swegle, IEEE Trans. Plasma Sci. PS-24, 1277 (1996); Phys. Fluids 25, 1282 (1982); 26, 1670 (1983).

${ }^{5}$ J. A. Swegle and E. Ott, Phys. Fluids 24, 1821 (1981).

${ }^{6}$ E. Ott and R. V. Lovelace, Appl. Phys. Lett. 27, 378 (1975).

${ }^{7}$ M. P. Desjarlais and R. N. Sudan, Phys. Fluids 29, 1746 (1986); 29, 1245 (1986); 30, 1536 (1987).
${ }^{8}$ K. D. Bergeron, Appl. Phys. Lett. 28, 306 (1976).

${ }^{9}$ C. W. Mendel, J. Appl. Phys. 50, 3830 (1979).

${ }^{10}$ P. A. Miller and C. W. Mendel, Jr., J. Appl. Phys. 61, 529 (1987).

${ }^{11}$ C. W. Mendel, D. B. Seidel, and S. A. Slutz, Phys. Fluids 26, 3628 (1983); C. W. Mendel, S. E. Rosenthal, and D. B. Seidel, Phys. Rev. A 45, 5854 (1992).

${ }^{12}$ N. A. Krall and S. E. Rosenthal, J. Appl. Phys. 70, 2542 (1991).

${ }^{13}$ J. M. Creedon, J. Appl. Phys. 48, 1070 (1977).

${ }^{14}$ T. R. Lockner and J. W. Poukey, J. Appl. Phys. 57, 2652 (1985)

${ }^{15}$ P. A. Lindsay and R. S. Goodell, J. Appl. Phys. 36, 411 (1965).

${ }^{16}$ T. J. Orzechowski and G. Bekefi, Phys. Fluids 19, 43 (1976); 22, 978 (1979).

${ }^{17}$ J. P. Verboncoeur and C. K. Birdsall, Phys. Plasmas 3, 712 (1996).

${ }^{18}$ V. P. Gopinath, J. P. Verboncoeur, and C. K. Birdsall, Phys. Plasmas 3, 2766 (1996); V. P. Gopinath and B. H. Vandenberg, ibid. 5, 261 (1998).

${ }^{19}$ B. W. Church and R. N. Sudan, Phys. Plasmas 3, 3809 (1996).

${ }^{20} \mathrm{C}$. K. Birdsall and W. B. Bridges, Electron Dynamics of Diode Regions (Academic, New York, 1966).

${ }^{21}$ R. B. Miller, An Introduction to the Physics of Intense Charged Particle Beams (Plenum, New York, 1982), Sec. 3.3.4.

${ }^{22}$ R. C. Davidson, Physics of Nonneutral Plasmas (Addison-Wesley, Redwood City, CA, 1990).

${ }^{23}$ J. P. Verboncoeur, M. V. Alves, V. Vahedi, and C. K. Birdsall, J. Comput. Phys. 104, 321 (1993).

${ }^{24}$ H. R. Jory and A. W. Trivelpiece, J. Appl. Phys. 40, 3924 (1969).

${ }^{25}$ B. Goplan, L. Ludeking, D. Smithe, and G. Warren, Comput. Phys. Commun. 87, 54 (1995).

${ }^{26}$ P. J. Christenson and Y. Y. Lau, Phys. Rev. Lett. 76, 3324 (1996).

${ }^{27}$ P. J. Christenson, D. P. Chernin, A. L. Garner, and Y. Y. Lau, Phys. Plasmas 3, 4455 (1996).

${ }^{28}$ A. L. Garner, Y. Y. Lau, and D. Chernin, Phys. Plasmas 5, 2447 (1998).

${ }^{29}$ J. C. Slater, Microwave Electronics (Van Nostrand, New York, 1950), Chap. 13.

${ }^{30}$ O. Buneman, in Crossed-Field Microwave Devices, edited by E. Okress (Academic, New York, 1961), Vol. 1, p. 209.

${ }^{31}$ H. L. McDowell, IEEE Trans. Plasma Sci. 30, 980 (2002).

${ }^{32}$ T. M. Antonsen, Jr. and E. Ott, Phys. Fluids 19, 52 (1976).

${ }^{33}$ T. M. Antonsen, Jr., E. Ott, C. L. Chang, and A. T. Drobot, Phys. Fluids 28, 2878 (1985).

${ }^{34}$ A. Valfells, D. W. Feldman, M. Virgo, P. G. O’Shea, and Y. Y. Lau, Phys. Plasmas 9, 2377 (2002).

${ }^{35}$ M. C. Clark, B. M. Marder, and L. D. Bacon, Appl. Phys. Lett. 52, 78 (1988).

${ }^{36}$ R. W. Lemke and M. C. Clark, J. Appl. Phys. 62, 3436 (1987).

${ }^{37}$ M. D. Haworth, G. Baca, J. Benford et al., IEEE Trans. Plasma Sci. 26, 312 (1998); K. J. Hendricks, J. Benford, J. Eastwood, M. Friedman, M. Haworth, R. W. Lemke, J. W. Luginsland, R. B. Miller, and J. Pasour, in High-Power Microwave Sources and Technologies, edited by R. J. Barker and E. Schamiloglu (IEEE, Piscataway, NJ, 2001), Chap. 3.

${ }^{38}$ D. Shiffler, G. Baca, T. Englert et al., IEEE Trans. Plasma Sci. 26, 304 (1998).

${ }^{39}$ F. J. Agee, W. L. Baker, and J. A. Gaudet, in High-Power Microwave Sources and Technologies, edited by R. J. Barker and E. Schamiloglu (IEEE, Piscataway, NJ, 2001), Chap. 2.

${ }^{40}$ Y. Y. Lau, P. J. Christenson, and D. P. Chernin, Phys. Fluids B 5, 4486 (1993).

${ }^{41}$ J. W. Luginsland, Y. Y. Lau, R. J. Umstatt, and J. J. Watrous, Phys. Plasmas 9, 2371 (2002); R. J. Umstattd and J. W. Luginsland, Phys. Rev. Lett. 87, 145002 (2001).

${ }^{42}$ J. W. Luginsland, Y. Y. Lau, and R. M. Gilgenbach, Phys. Rev. Lett. 77, 4668 (1996); Y. Y. Lau, ibid. 87, 278301 (2001).

${ }^{43}$ F. Hegeler, M. Friedman, M. C. Myers, J. D. Sethian, and S. B. Swanekamp, Phys. Plasmas 9, 4309 (2002). 